\title{
Central Nervous System and Cardiovascular Diseases
}

\author{
Maurizio Arduini
}

\begin{abstract}
Children with congenital heart diseases (CHD) are at increased risk of neurodevelopmental delay (NDD). In fetuses with CHD, blood streaming in the brain may be partially deoxygenated. Signs of cerebrovascular autoregulation comparable to the "brain-sparing effect" in growth-restricted fetuses may be shown in fetuses with CHD depending on the type of heart disease. This effect appears to be predominantly present in fetuses with left-side obstructive lesions (LSOL). During prenatal counseling, it is recommended to mention the possibility of an increased risk of NDD. The severity of this impairment varies from the type of $\mathrm{CHD}$, being highest up to 40 to $45 \%$ in lesions with univentricular heart hemodynamics, such as hypoplastic left heart syndrome (HLHS). The prenatal life plays an important role in determining postnatal neurodevelopmental functions, but the severity of NDD in fetuses with CHD cannot be predicted prenatally.
\end{abstract}

Keywords: Brain sparing effect, Congenital heart diseases, Neurodevelopmental delay.

How to cite this article: Arduini M. Central Nervous System and Cardiovascular Diseases. Donald School J Ultrasound Obstet Gynecol 2017;11(4):362-366.

Source of support: Nil

Conflict of interest: None

\section{INTRODUCTION}

Congenital heart disease is one of the most common types of congenital malformations, affecting between 6 and 8 per 1,000 newborns, and thus remains the leading cause of death in children with congenital malformations. ${ }^{1}$

As recently as the 1960 s, only $20 \%$ of neonates with critical CHD survived to adulthood. Nowadays, the possibility of a prenatal diagnosis of $\mathrm{CHD}^{2,3}$ and the development of new surgical techniques with postoperative intensive care have improved the long-term survival rate of patients suffering from CHD. Indeed, for some major diseases, such as the transposition of the great arteries, survival has reached $90 \%$ after a follow-up of 10 years. ${ }^{4}$ Unfortunately, though, these important lifesaving improvements have not gone hand-in-hand with a decrease in neonatal morbidity.

\section{Consultant}

Department of Obstetrics and Gynecology, Fetal-Maternal Medicine Hospital, Foligno-Perugia, Italy

Corresponding Author: Maurizio Arduini, Consultant Department of Obstetrics and Gynecology, Fetal-Maternal Medicine Hospital, Foligno-Perugia, Italy, Phone: 3476045328 e-mail: maoard@libero.it
In particular, neurodevelopmental disability is now recognized as the most common long-term complication of critical CHD. It is also the complication with the most negative impact on quality of life, academic performance, and opportunity for independence as an adult.

The increasing supportive evidence in this field has led to the publication of the following official scientific statement by the American Heart Association: "Children with CHD are at increased risk of developmental disorder or disabilities or developmental delay." ${ }^{5}$

These neurodevelopmental disorders were assumed to be the result of perioperative conditions, resulting in cerebral hypoxia and thromboembolic events. ${ }^{6}$

Recent studies have demonstrated signs of abnormal neurological development already present at birth, prior to surgery.

Prenatal ultrasound and RMI describe abnormal or delayed sulcation, reduced brain biometry and volumes, and abnormal brain biochemistry, in fetuses with some forms of CHD. ${ }^{7-11}$

This suggests that the prenatal environment plays an important role in determining postnatal neurodevelopmental functions. This is in contrast with the traditional concept which attributes adverse neurodevelopmental outcomes to postnatal events, such as perinatal hypoxia and intraoperative damage. ${ }^{6}$

The association between CHD and NDD remains poorly understood, being almost certainly multifactorial.

\section{CEREBRAL BLOOD FLOW AND CHD: BRAIN-SPARING EFFECT}

In healthy fetuses, circulation directs oxygenated blood from the left ventricle and the ascending aorta to the fetal brain, and deoxygenated blood to the placenta (Fig. 1).

In fetuses with CHD, blood to the brain may be partially deoxygenated, depending on the type of heart disease (Figs 2 and 3).

The normal development of the fetal CNS is extremely complex. During fetal life various unfavorable events can occur influencing regular development. Certainly, hypoxia is among the main causes of neurological damage that can occur during fetal life.

In the past decade, a number of prenatal Doppler studies have investigated whether fetuses with cardiac defects associated with relative cerebral hypoxemia show signs of cerebrovascular autoregulation, comparable to the "brain-sparing effect" in growth-restricted fetuses. 


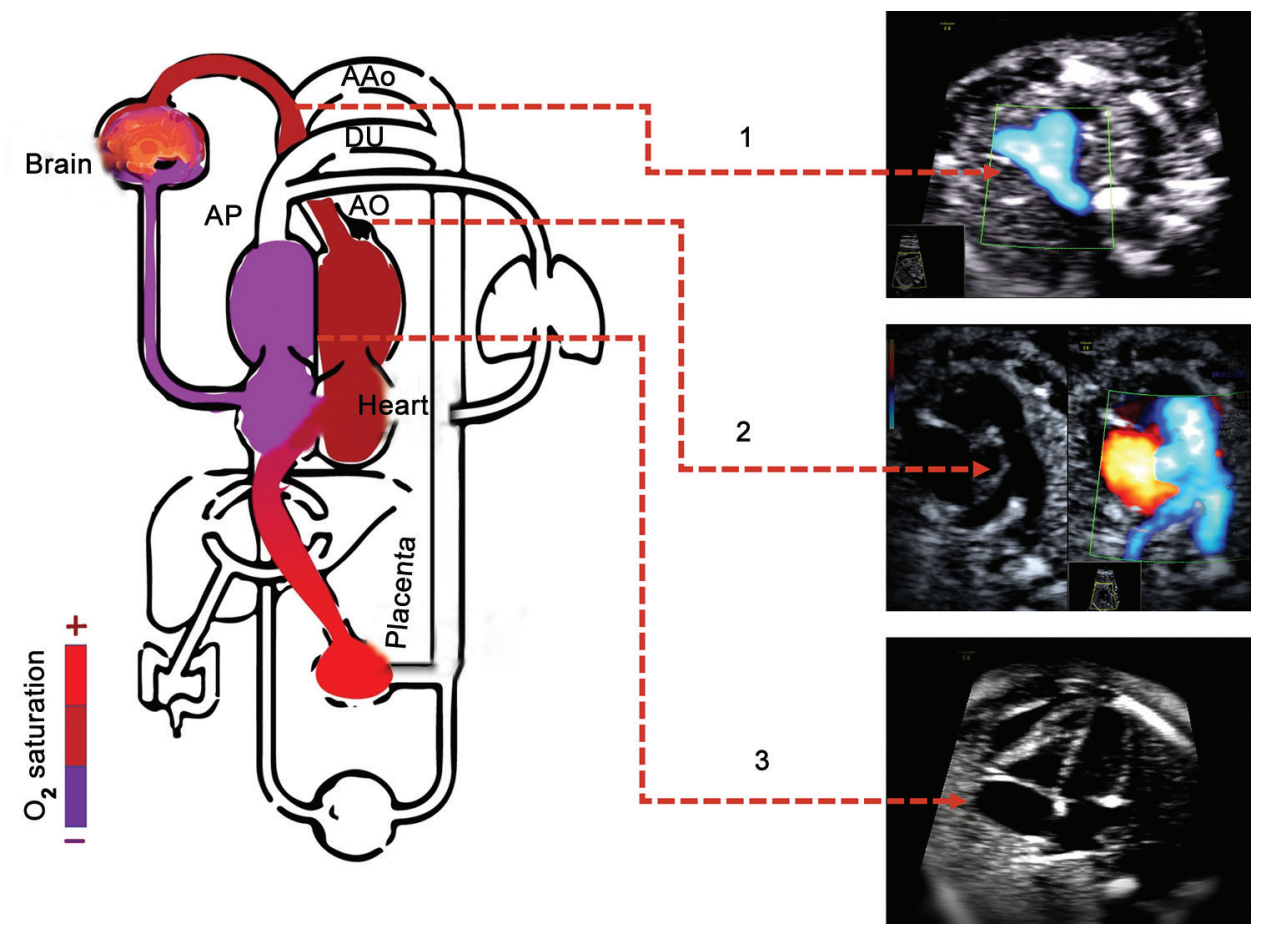

Fig. 1: In the normal fetal circulation, streaming of very oxygenated blood from the placenta directly to the fetal cerebral circulation via the ductus venosus and foramen ovale. Numbers 1 to 3 show the ultrasound scan section side. (1) Three-vessel view: Aortic and ductus arteriosus arches flow in the same direction, anterograde flow. (2) Short axis: Normal diameter of aortic and pulmonary artery. (3) Apical four-chamber view (AAo: Aortic arch; DU: Ductus; AO: Aorta; AP: Pulmonary artery)

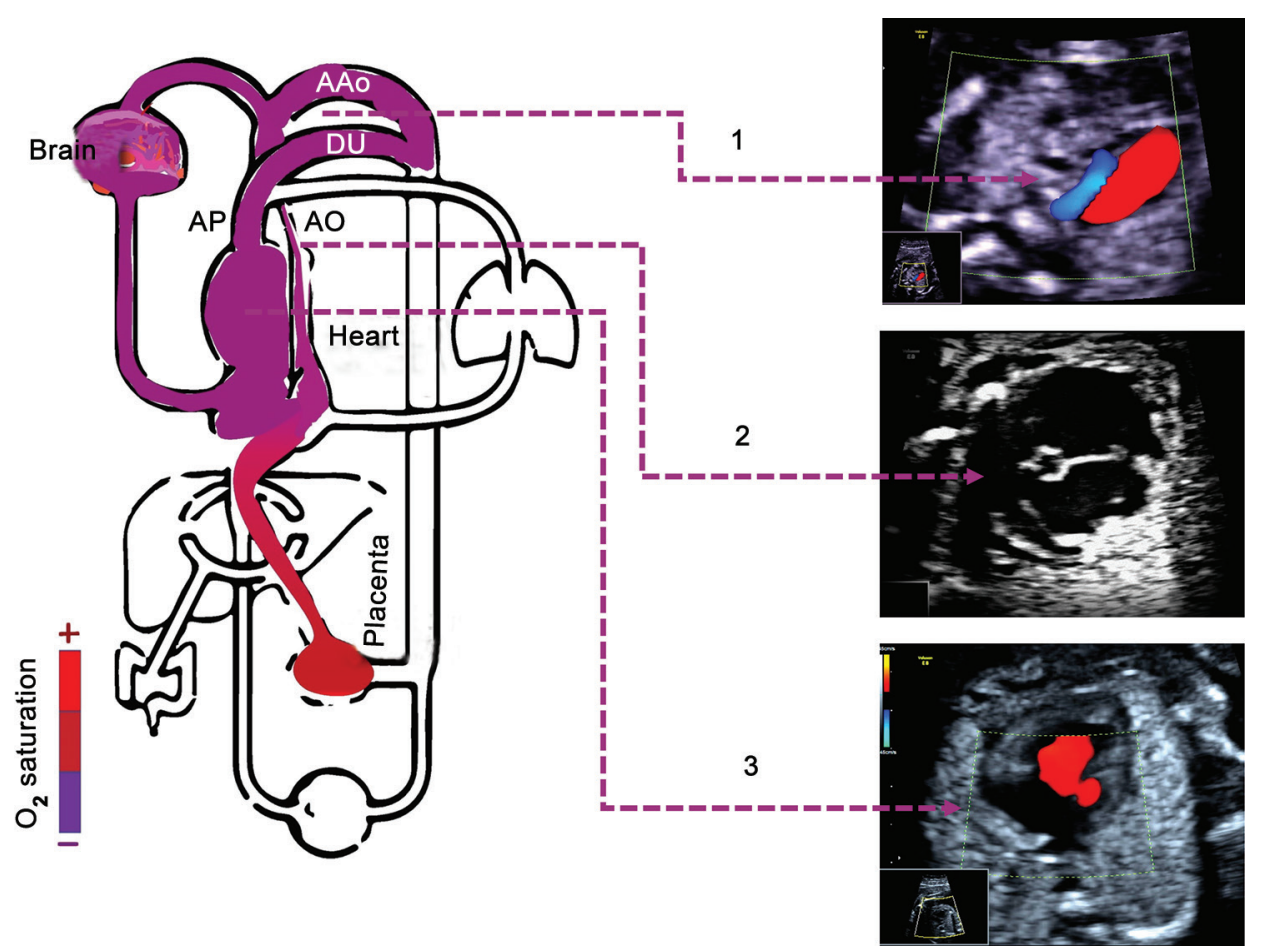

Fig. 2: In LSOL, oxygenated and deoxygenated blood is mixed in the right ventricle. Very deoxygenated blood flows in the fetal cerebral circulation via ductus arteriosus and retrograde flow of aortic arch. (1) Three-vessel view with retrograde flow in the aortic arch. $(2,3)$ Short axis and apical four-chamber view: Hypoplastic aortic annulus and virtual left ventricular heart "hypoplastic left heart" (AAo: Aortic arch; DU: Ductus; AO: Aorta; AP: Pulmonary artery) 


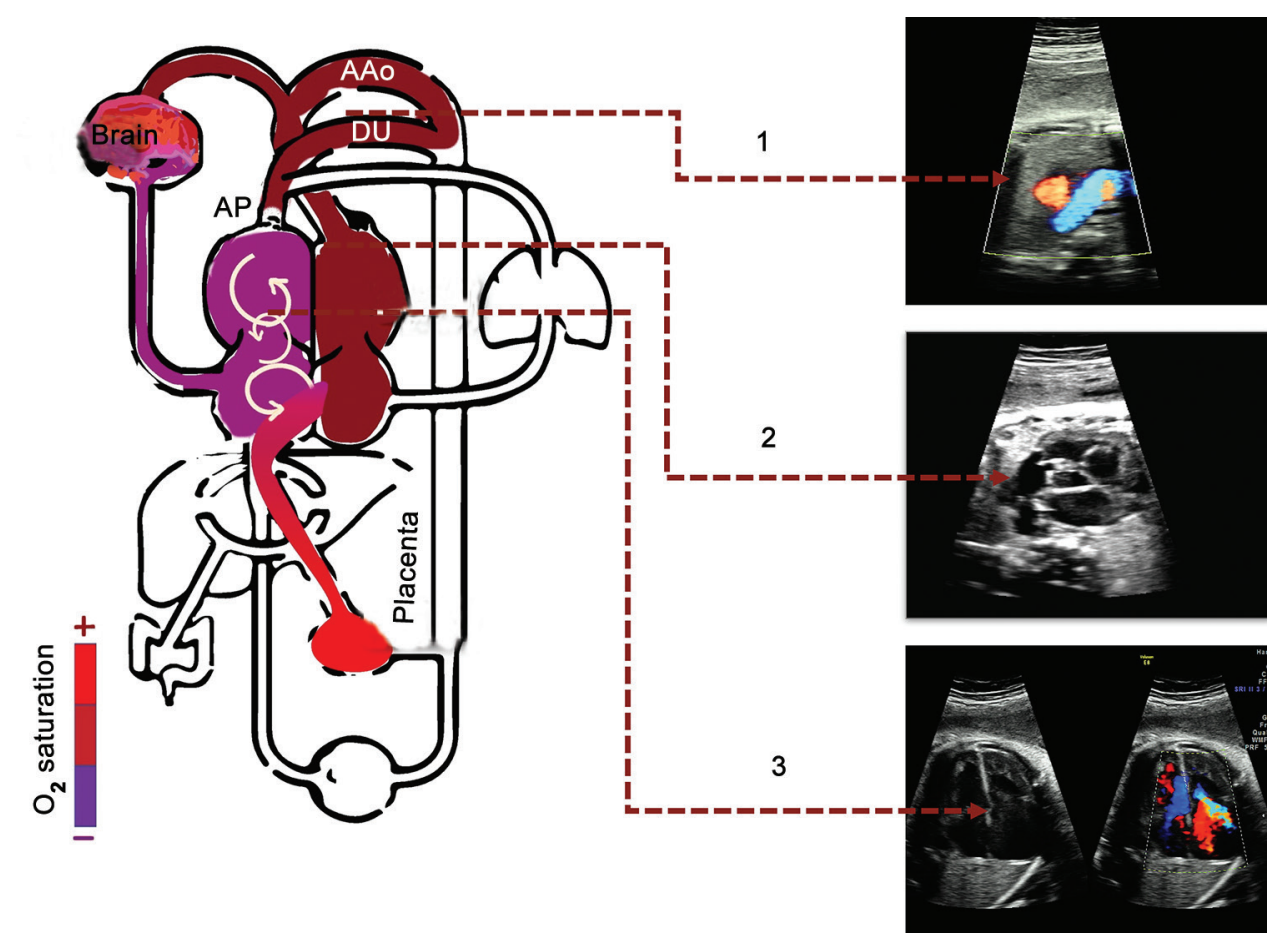

Fig. 3: In RSOL, oxygenated blood from the placenta and deoxygenated blood from right ventricle is mixed in the fetal heart. Deoxygenated blood flows in the fetal cerebral circulation via left ventricle and aortic arch. (1) Three-vessel view with retrograde flow in the ductus arteriosus. $(2,3)$ Short axis and apical four-chamber view: Pulmonary atresia, hypertrophic right ventricle, and regurgitation of tricuspid valve (AAo: Aortic arch; DU: Ductus; AO: Aorta; AP: Pulmonary artery)

According to a previous study carried out by D'Onofrio, fetuses with CHD have a higher incidence of cerebral/ placental resistance ratio $(\mathrm{CPR})<1$ vs healthy fetuses controls. Said study assessed CPR as a measure of cerebral autoregulation and brain-sparing effect. Fetuses with HLHS are the most affected. ${ }^{12}$

Similar results were obtained by Berg Modena and Kaltman: Different types of CHD have different impacts on fetal cerebrovascular blood flow distribution. However, only fetuses with HLHS showed significant alterations detectable by evaluating middle cerebral artery (MCA) Doppler indices. ${ }^{13-15}$

Our experience ${ }^{16}$ confirmed a significantly lower cerebrovascular resistance in CHD fetuses when compared with the control group. This change of cerebral hemodynamics was also better identified analyzing the CPR. When the heart diseases were considered individually, the lowest values of the CPR ratio were found in left heart diseases.

A recent review and a meta-analysis show that fetuses with CHD demonstrate signs of impaired brain development, identified either with fetal ultrasound (US) or with magnetic resonance imaging (MRI). The most studied parameter is the MCA, which shows a slightly increased end-diastolic blood flow in CHD fetuses. This effect appears to be present predominantly in LSOL. The MCA flow appears not to be altered in right-side obstructive lesions (RSOL). ${ }^{17}$
In fetuses with HLHS it seems plausible that the flow restriction to the carotid arteries, in cases of reversed aortic arch flow, can induce vasodilatation in the cerebral circulatory system to facilitate and secure the transport of oxygen and nutrients (glucose) (Fig. 2). Fetuses with a univentricular heart show a significant reduction of the third-trimester biometrical head parameter. ${ }^{17}$

Similarly, in our experience, fetuses with CHD revealed head circumference (HC) values significantly lower than in the normal population. On the contrary, abdominal circumference (AC) measurements appeared similar in the two groups examined, revealing more evident differences compared with the HC/ AC ratio. ${ }^{16}$

Moreover, the type of $\mathrm{CHD}$ with the lowest value of $\mathrm{CPR}$ also had lower values of $\mathrm{HC} / \mathrm{AC}$, corroborating the hypothesis of a chronic deficit of cerebral oxygen. ${ }^{16}$

The recent use of RMI has confirmed that brain volume and metabolism are abnormal in third-trimester fetuses with some forms of complex CHD, particularly those with HLHS. It has also evidenced a reduced flow through the systemic ventricle or antegrade around the aortic arch. The cerebral $\mathrm{N}$-acetyl aspartate/choline (NAA/Cho) ratio was used to measure the cerebral lactate. The NAA/Cho increased progressively over the third trimester in normal fetuses, but the rate of said rise was significantly slower $(\mathrm{p}<0.001)$ in CHD fetuses. ${ }^{9}$ 
Sun et $\mathrm{al}^{7}$ support a direct link between reduced cerebral oxygenation and impaired brain growth in fetuses with CHD. They suggest the possibility that in utero brain development could be improved with maternal oxygen therapy.

In a recent systematic review, Khalil analyzed the correlation of the altered brain circulation in fetuses with $\mathrm{CHD}$ and the prevalence of prenatal brain abnormalities.

Unexpectedly, fetuses with CHD, especially HLHS, present a high incidence ( $28 \%$ ) of fetal brain malformation. The most commonly reported abnormality was ventriculomegaly $(8.6 \%)$. Other abnormalities included malformation or delay of cortical development, vermian hypoplasia, agenesis of the corpus callosum, holoprosencephaly, cerebellar hypoplasia, and ventricular bleeding. A possible explanation for this association could be in utero altered cerebral circulation in fetuses with $\mathrm{CHD} .{ }^{18}$

\section{BRAIN-SPARING EFFECT AND NEURODEVELOPMENTAL OUTCOME}

The correlation of cerebrovascular resistance and CHD has been well studied showing the presence of brainsparing effect in fetuses with LSOL. It is very important to investigate the association of cerebrovascular resistance with neurodevelopment outcomes.

Mental and psychomotor developmental indices (PDI) were assessed at 14 months and correlated with MCA flow.

Williams et $\mathrm{al}^{19}$ reported that lower MCA-pulsatility index (PI), and thus lower cerebrovascular resistance, was associated with a higher 14-month neurodevelopmental score in fetuses with HLHS.

Middle cerebral artery PI z-score is inversely correlated with PDI. ${ }^{20}$

Surprisingly, this relationship is opposite to that observed with advanced intrauterine growth retardation (IUGR). Moreover, it may represent a unique ability of these CHD fetuses to compensate for a diminished cerebral oxygen delivery.

One possible explanation is the difference in underlying pathophysiology. Systemic hypoxia in IUGR fetuses may be more severe than what is needed in single ventricle fetuses. Therefore, the autoregulatory response of cerebral vasodilation may be sufficient and even adaptive. $^{19}$

In a subsequent paper, the author partitioned data into specific gestational periods to evaluate changes over time and to determine whether any specific period in gestation was more highly associated with developmental outcomes.

Examining four gestational periods (20-23; 24-29; 30-33; >34 weeks), the mean PI z-score was significantly different from zero at all gestational periods. The most negative difference of PI z-score in middle cerebral artery (MCA) was identified in the last gestational period. Interestingly, the rate of change of the MCA PI z-score was not statistically significant from 20 to 33 weeks. ${ }^{20}$

In this group of fetuses, we were unable to show a correlation between MCA PI z-score at a specific gestational period and neurodevelopmental outcomes.

\section{CONCLUSION AND COUNSELING}

Congenital heart disease is one of the most common types of congenital malformations. An estimated $85 \%$ of children diagnosed with CHD survive into adulthood. ${ }^{2}$

The reduction in the mortality rate, determined by new surgical technologies, has shifted researchers' attention toward an attempt to understand, and possibly reduce, neonatal morbidity, as a result of altered neurological development in fetuses affected by $\mathrm{CHD} .{ }^{16}$

The prevalence and severity of NDD increase with the complexity of CHD. ${ }^{5}$

It is estimated that $70 \%$ of children with HLHS suffer from neurodevelopmental deficits. ${ }^{5}$

Fetuses with isolated severe CHD demonstrate signs of impaired fetal cerebral development, identified by fetal US or Magnetic Resonance Imaging (MRI). ${ }^{9}$

A decrease in cerebrovascular resistance, comparable to the "brain-sparing effect" in growth-restricted fetuses, is present in fetuses with CHD. Moreover, it may represent a marker of cerebral hypoxemia due to intracardiac mixing of oxygenated and deoxygenated blood. ${ }^{9}$

This effect appears to be predominantly present in fetuses with LSOL. The MCA flow appears not to be altered in RSOL (Figs 1 and 2).

A decrease in cerebral artery resistance in fetuses with HLHS is identified mainly in the third trimester of pregnancy, although initial changes may be already evident from the 20th week of gestation. ${ }^{20}$

The relationship between MCA PI and neurodevelopment is controversial. Among fetuses with LSOL, lower cerebrovascular resistance can be associated with higher neurodevelopment scores. This relationship is opposite to that observed with advanced IUGR and may represent a unique ability of these $\mathrm{CHD}$ fetuses to compensate for diminished cerebral oxygen delivery.

The association between CHD and NDD is certainly multifactorial. Indeed, the prenatal life plays an important role in determining postnatal neurodevelopmental functions.

The increasing supportive evidence in this field has led to the publication of the following official scientific statement by the International Society of Ultrasound in 
Obstetrics and Gynecology (ISUOG): "Fetuses with hypoplastic left heart (HLH) show an increased risk of NDD, regardless of surgery. For all other $\mathrm{CHD}$, current evidence should be supported by further studies of children with prenatal diagnosis and optimal perinatal management before providing the same type of counseling as for those with an univentricular circulation."

During prenatal counseling in fetuses with CHD, it is recommended to mention the possibility of an increased risk of NDD.

The ISUOG suggests that the following statement may be helpful during counseling: “The majority of fetuses/ neonates with isolated CHD do well. However, there is evidence that some have a degree of NDD, which cannot be predicted antenatally. The severity of this impairment varies from individual to individual, and the likely incidence varies with the type of $\mathrm{CHD}$, being highest (up to $40-45 \%$ ) in lesions with univentricular heart hemodynamics, such as HLHS." 21

\section{REFERENCES}

1. Hoffman JI, Kaplan S. The incidence of congenital heart disease. J Am Coll Cardiol 2002 Jun;39(12):1890-1900.

2. Bakiler AR, Ozer EA, Kanik A, Kanit H, Aktas FN. Accuracy of prenatal diagnosis of congenital heart disease with fetal echocardiography. Fetal Diagn Ther 2007;22(4):241-244.

3. Gottliebson WM, Border WL, Franklin CM, Meyer RA, MichelFelder EC. Accuracy of fetal echocardiography: a cardiac segment-specific analysis. Ultrasound Obstet Gynecol 2006 Jul;28(1):15-21.

4. De Koning WB, Van Osch-Gevers M, Harkel AD, Van Domburg RT, Spijkerboer AW, Utens EM, Bogers AJ, Helbing WA. Follow-up outcomes 10 years after arterial switch operation for transposition of the great arteries: comparison of cardiological health status and health-related quality of life to those of a normal reference population. Eur J Pediatr 2008 Sep;167(9):995-1004.

5. Marino BS, Lipkin PH, Newburger JW, Peacock G, Gerdes M, Gaynor JW, Mussatto KA, Uzark K, Goldberg CS, Johnson WH Jr, et al. Neurodevelopmental outcomes in children with congenital heart disease: evaluation and management: a scientific statement from the American Heart Association. Circulation 2012 Aug 28;126(9):1143-1172.

6. Plessis AJ. Mechanisms of brain injury during infant cardiac surgery. Semin Pediatr Neurol 1999 Mar;6(1):32-47.

7. Sun L, Macgowan CK, Sled JG, Yoo SJ, Manlhiot C, Porayette P, Grosse-Wortmann L, Jaeggi E, McCrindle BW, Kingdom J, et al. Reduced fetal cerebral oxygen consumption is associated with smaller brain size in fetuses with congenital heart disease. Circulation 2015 Apr 14;131(15):1313-1323.

8. Clouchoux C, du Plessis AJ, Bouyssi-Kobar M, Tworetzky W, McElhinney DB, Brown DW, Gholipour A, Kudelski D, Warfield SK, McCarter RJ, et al. Delayed cortical development in fetuses with complex congenital heart disease. Cereb Cortex 2012 Dec;23(12):2932-2943.
9. Limperopoulos C, Tworetzky W, McElhinney DB, Newburger JW, Brown DW, Robertson RL Jr, Guizard N, McGrath E, Geva J, Annese D, et al. Brain volume and metabolism in foetuses with congenital heart disease: evaluation with quantitative magnetic resonance imaging and spectroscopy. Circulation 2010 Jan 5;121(1):26-33.

10. Mlczoch E, Brugger P, Ulm B, Novak A, Frantal S, Prayer D, Salzer-Muhar U. Structural congenital brain disease in congenital heart disease: results from a fetal MRI program. Eur J Paediatr Neurol 2013 Mar;17(2):153-160.

11. Donofrio MT, Duplessis AJ, Limperopoulos C. Impact of congenital heart disease on fetal brain development and injury. Curr Opin Pediatr 2011 Oct;23(5):502-511.

12. Donofrio MT, Bremer YA, Schieken RM, Gennings $C$, Morton LD, Eidem BW, Cetta F, Falkensammer CB, Huhta JC, Kleinman CS. Autoregulation of cerebral blood flow in fetuses with congenital heart disease: the brain sparing effect. Pediatr Cardiol 2003 Sep-Oct;24(5):436-443.

13. Berg C, Gembruch O, Gembruch U, Eipel A. Doppler indices of the middle cerebral artery in fetuses with cardiac defects theoretically associated with impaired cerebral oxygen delivery in utero: is there a brain-sparing effect? Ultrasound Obstet Gynecol 2009 Dec;34(6):666-672.

14. Modena A, Horan C, Visintine J, Chanthasenanont A, Wood D, Weiner $\mathrm{S}$. Fetuses with congenital heart disease demonstrate signs of decreased cerebral impedance. Am J Obstet Gynecol 2006 Sep;195(3):706-710.

15. Kaltman JR, Di H, Tian Z, Rychik J. Impact of congenital heart disease on cerebrovascular blood flow dynamics in the fetus. Ultrasound Obstet Gynecol 2005 Jan;25(1):32-36.

16. Arduini M, Rosati P, Caforio L, Guariglia L, Clerici G, Di Renzo GC, Scambia G. Cerebral blood flow autoregulation and congenital heart disease: possible causes of abnormal prenatal neurologic development. J Matern Fetal Neonatal Med 2011 Oct;24(10):1208-1211.

17. Jansen FA, Everwijn SM, Scheepjens R, Stijnen T, PeetersScholte CM, van Lith JM, Haak MC. Fetal brain imaging in isolated congenital heart defects-a systematic review and meta-analysis. Prenat Diagn 2016 Jul;36(7):601-613.

18. Khalil A, Suff N, Thilaganathan B, Hurrell A, Cooper D, Carvalho JS. Brain abnormalities and neurodevelopmental delay in congenital heart disease: systematic review and metaanalysis. Ultrasound Obstet Gynecol 2014 Jan;43(1):14-24.

19. Williams IA, Fifer C, Jaeggi E, Levine JC, Michelfelder EC, Szwast AL. The association of fetal cerebrovascular resistance with early neurodevelopment in single ventricle congenital heart disease. Am Heart J 2013 Apr;165(4):544-550.

20. Hahn E, Szwast A, Cnota J, Levine JC, Fifer CG, Jaeggi E, Andrews H, Williams IA. Association between fetal growth, cerebral blood flow and neurodevelopmental outcome in univentricular fetuses. Ultrasound Obstet Gynecol 2016 Apr;47(4):460-465.

21. Paladini D, Alfirevic Z, Carvalho JS, Khalil A, Malinger G, Martinez JM, Rychik J, Ville Y, Gardiner H, ISUOG Clinical Standards Committee. ISUOG consensus statement on current understanding of the association of neurodevelopmental delay and congenital heart disease: impact on prenatal counseling. Ultrasound Obstet Gynecol 2017 Feb;49(2):287-288. 